\title{
PREPARATION AND IN VITRO TEST OF APATITE FILMS ONTO TITANIUM BY SPUTTERING FROM CALCIUM PHOSPHATE POWDER TARGETS
}

\author{
JUN-ICHI HAMAGAMI ${ }^{1}$, DAISUKE KOKUBU ${ }^{1}$, KIYOSHI KANAMURA ${ }^{1}$, \\ TAKAO UMEGAKI ${ }^{1}$ and KIMIHIRO YAMASHITA ${ }^{2}$
}

${ }^{1}$ Department of Applied Chemistry, Graduate School of Engineering, Tokyo Metropolitan University, 1-1 Minami-Osawa, Hachioji, Tokyo 192-0397, Japan

${ }^{2}$ Institute of Biomaterials and Bioengineering, Tokyo Medical and Dental University, 2-3-10 Kanda-Surugadai, Chiyoda, Tokyo 101-0062, Japan

\begin{abstract}
Polycrystalline hydroxyapatite $\left(\mathrm{Ca}_{10}\left(\mathrm{PO}_{4}\right)_{6}(\mathrm{OH})_{2}\right.$, HAp) coatings were prepared by rf-sputtering combined with hydrothermal annealing, and then their biocompatibility through in vitro biological test was examined. Amorphous calcium phosphate thin films were deposited onto titanium metal plates by an rf magnetron sputtering without a substrate heating. The targets for sputtering were three kinds of compressed powder of dicalcium phosphate (DCP), $\alpha$-tetracalcium phosphate $(\alpha$-TCP), and hydroxyapatite (HAp). In order to crystallize the amorphous coatings, hydrothermal annealing was performed using an autoclave at $200^{\circ} \mathrm{C}$ for $24 \mathrm{~h}$ under a saturated water vapor pressure. Biocompatibility of the postannealed specimens was examined by in vitro test using a simulated body fluid (SBF) with $\mathrm{pH} 7.4$ at $36.5^{\circ} \mathrm{C}$. Surface morphologies and $\mathrm{Ca} / \mathrm{P}$ ratios of the films depend on the target composition for sputtering. Since SEM observation shows bonelike crystal growth on the surfaces of the films after soaking in an SBF, all the specimens have good biocompatibility.
\end{abstract}

\section{INTRODUCTION}

A radio-frequency (rf) magnetron sputtering combined with annealing is one of the practical coating methods of calcium-hydroxyapatite $\left(\mathrm{Ca}_{10}\left(\mathrm{PO}_{4}\right)_{6}(\mathrm{OH})_{2}\right.$, HAp) onto metals or ceramics with excellent mechanical properties for implant application. ${ }^{1-10}$ A sputtering method has advantages of thickness uniformity of coatings, and high adhesion strength between the coatings and the substrates. Biocompatibility of the sputtered coatings was also confirmed by in vivo test using rabbit. $^{10}$ We have investigated preparation of single phase of HAp thin films onto ceramics or 
metals through rf magnetron sputtering for biomedical use. ${ }^{1-9}$ It is well known that the composition ( $\mathrm{Ca} / \mathrm{P}$ ratio) of the coatings are different from that of the targets for sputtering. We have succeeded the formation of single-phase HAp coatings from the sputtering targets of the calcium phosphate glasses with much lower than a $\mathrm{Ca} / \mathrm{P}$ ratio of a stoichiometric HAp followed by annealing treatment. ${ }^{2,6}$ Recently, we have also demonstrated a single-phase HAp thin films were successfully deposited on titanium metal using calcium phosphate powders with $\mathrm{Ca} / \mathrm{P}$ ratios of 1.0 to 1.67 , combined with a hydrothermal annealing process at low temperatures. ${ }^{9}$

In this work, hydroxyapatite coatings were deposited onto titanium plates by an if magnetron sputtering from the powder targets of dicalcium phosphate, $\alpha$-tricalcium phosphate, and hydroxyapatite, combined with the low-temperature hydrothermal annealing treatment, and then their biological performance was in vitro tested by using a simulated body fluid.

\section{EXPERIMENTAL PREOCEDURE}

Hydroxyapatite thin films were prepared by rf-sputtering combined with annealing. First precursory thin films were deposited on titanium metal plates by using an rf magnetron sputtering apparatus (SPK-301, Tokki). The targets for sputtering were three kinds of compressed bodies of dicalcium phosphate (DCP), $\alpha$-tricalcium phosphate ( $\alpha$-TCP), and hydroxyapatite (HAp) commercial powders (Taihei Chemical Ind. Co.). Sputtering was carried out in an argon atmosphere of $0.67 \mathrm{~Pa}$ by applying if input power of $2.6 \mathrm{~W} \mathrm{~cm}^{-2}$. The substrates were not intentionally heated, but the substrate temperature increased up to $150^{\circ} \mathrm{C}$ during deposition. Subsequently, the as-sputtered films were annealed hydrothermally in order to obtain a high crystalline HAp. Postannealing was conducted at $200^{\circ} \mathrm{C}$ for $24 \mathrm{~h}$ under a saturated water vapor pressure in an autoclave. After the hydrothermal annealing, all the specimens were mildly washed with distilled water and then dried at room temperature.

In vitro biological evaluation of the specimens was carried out using a simulated body fluid (SBF) with ion concentrations approximately equal to those of human blood plasma. ${ }^{11}$ All the postannealed films were immersed in an $\mathrm{SBF}$ with $\mathrm{pH}=7.4$ at $36.5^{\circ} \mathrm{C}$ for prescribed time. After soaking in an SBF, the specimens were observed by a scanning electron microscopy (SEM) to confirm the formation of the grown crystals and/or layers on their surfaces.

The crystal structures of the films were examined by X-ray diffraction (XRD) measurements with $\mathrm{Cu}-\mathrm{K}_{\alpha}$ radiation. Fourier Transformed Infrared (FT-IR) spectra for the films were measured from $4000 \mathrm{~cm}^{-1}$ to $400 \mathrm{~cm}^{-1}$ in order to evaluate the $\mathrm{PO}_{4}{ }^{3-}$ and $\mathrm{OH}^{-}$ion groups in the films. The surface morphologies and the $\mathrm{Ca} / \mathrm{P}$ ratios of the specimens were investigated by SEM equipped with energy dispersive $\mathrm{X}$-ray spectroscopy (EDS). 


\section{RESULTS AND DISCUSSION}

Since as-sputtered films were confirmed amorphous from XRD patterns, the hydrothermal annealing was carried out at $200^{\circ} \mathrm{C}$ for $24 \mathrm{~h}$. Figure 1 shows the XRD patterns of the postannealed films onto titanium substrates sputtered from powder targets with a $\mathrm{Ca} / \mathrm{P}$ ratio of (a) 1.0, (b) 1.5, and (c) 1.67. The diffraction peaks observed for all the specimens were assigned to those of

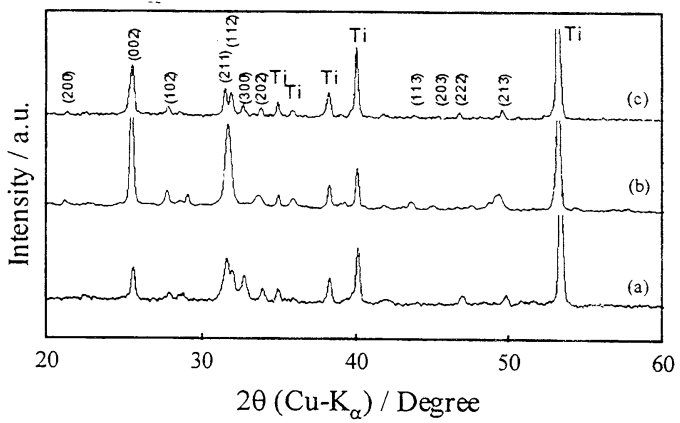

FIGURE 1. XRD patterns of the postannealed thin films sputtered from powder targets with a $\mathrm{Ca} / \mathrm{P}$ ratio of (a) 1.0 , (b) 1.5 , and (c) 1.67 .

$\mathrm{Ca}_{5}\left(\mathrm{PO}_{4}\right)_{3} \mathrm{OH}$, HAp (JCPDS \#9-432) and titanium substrate, independent of the composition of the targets for sputtering. Any other related calcium phosphates such as tricalcium phosphate, tetracalcium phosphate, and calcium oxides were not detected in the postannealed films.

FT-IR measurements of the specimens were carried out in order to study the local structure concerning the characteristic ion groups of $\mathrm{PO}_{4}{ }^{3-}$ and $\mathrm{OH}^{-}$in the HAp lattice. Figure 2 shows the FT-IR spectra of the postannealed films sputtered from powder targets with a Ca/P ratio of (a) 1.0 and (b) 1.67, respectively. The IR absorption peaks at around 1090, 1035, 960, 602 and $568 \mathrm{~cm}^{-1}$ were assigned to the ion groups of $\mathrm{PO}_{4}{ }^{3-}$ in $\mathrm{HAp}$ structure. Furthermore, the peaks at 3571 and 628 $\mathrm{cm}^{-1}$ due to the $\mathrm{OH}^{-}$ions in HAp were also detected. All the present films sputtered from target compositions of a $\mathrm{Ca} / \mathrm{P}$ ratio of $1.0,1.5$, and 1.67 were confirmed as a single phase of a polycrystalline HAp from the results of XRD and FT-IR analyses.
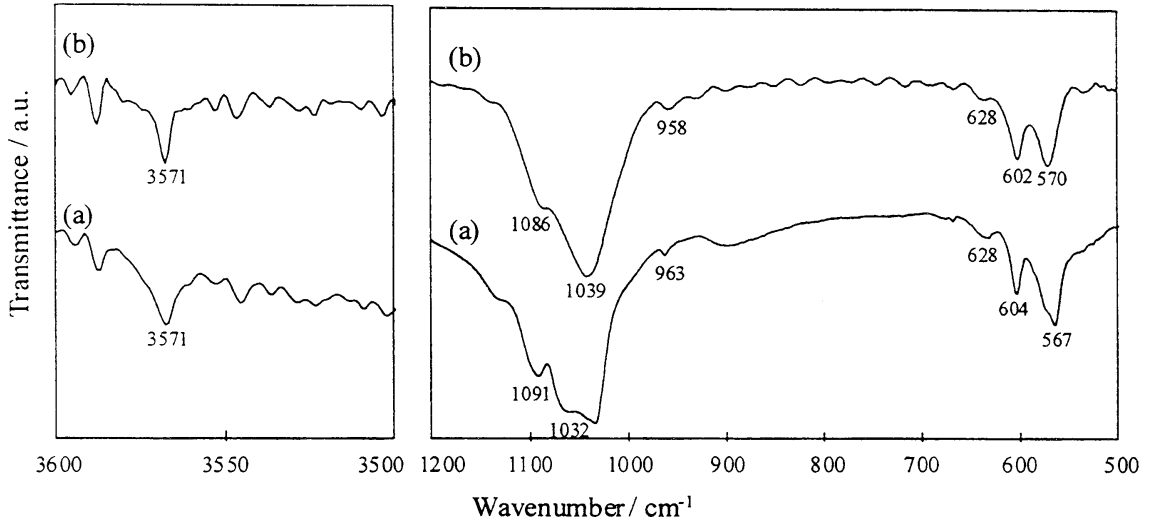

FIGURE 2. FT-IR spectra of the postannealed specimens sputtered from powder targets with a $\mathrm{Ca} / \mathrm{P}$ ratio of (a) 1.0 and (b) 1.67.

All the as-sputtered films had smooth surfaces by SEM observation. On the other hand, the surface morphologies of the postannealed films were different from those of the as-sputtered 
films. Figure 3 shows the SEM photographs of the surfaces of the postannealed specimens onto titanium sputtered from powder targets of a $\mathrm{Ca} / \mathrm{P}$ ratio of $1.0,1.5$ and 1.67 . In the case of $\mathrm{Ca} / \mathrm{P}=$ 1.0 , the needle-like crystals are covered with titanium substrate. Another cases of $\mathrm{Ca} / \mathrm{P}=1.5$ and 1.67 , the surface morphologies are homogeneous.

Figure 4 shows SEM micrographs of the surfaces of the postannealed films, which were sputtered from powder target composition with a Ca/P ratio of (a) 1.0, (b) 1.5, and (c) 1.67, after soaking in an SBF for (a) 20 days, (b) and (c) 1 day. Since crystal growths on their surfaces were clearly observed, all the specimens have good biocompatibility. The crystal growth rate was dependent upon the target composition for sputtering; it increases with an increase in $\mathrm{Ca} / \mathrm{P}$ ratio of the target for sputtering. No crystal growth of the specimens using target with $\mathrm{Ca} / \mathrm{P}=1.0$ was observed after soaking in an SBF within 3 days. When the target compositions were $\mathrm{Ca} / \mathrm{P}=1.5$ and 1.67, the crystal growth layers were clearly observed the specimens soaked in an SBF even for 1 day. The grown layers were analyzed by XRD, FT-IR, and EDS. From the results of XRD and EDS analysis, the grown layers were apatitic calcium phosphate crystals containing a small amount of Mg. FT-IR spectra show the presence of the carbonate ions. These analytical results indicate the formation of bonelike crystals of partially carbonated calcium hydroxyapatite.
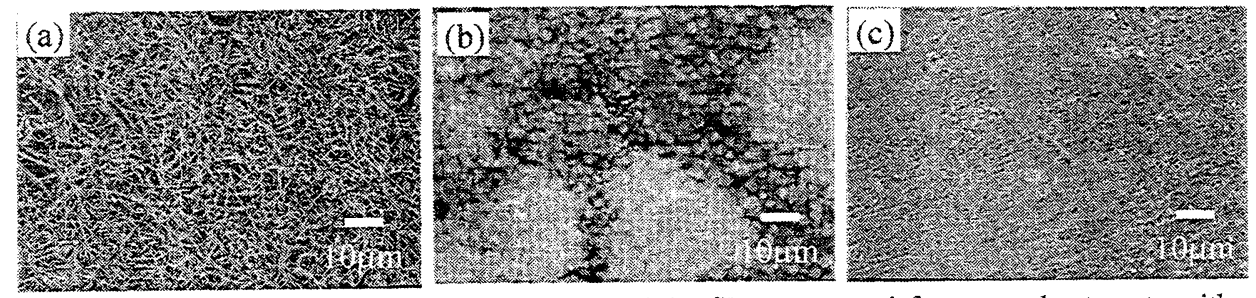

FIGURE 3. SEM photographs of the surfaces of the films sputtered from powder targets with a $\mathrm{Ca} / \mathrm{P}$ ratio of (a) 1.0 , (b) 1.5 , and (c) 1.67 , followed by the hydrothermal annealing at $200^{\circ} \mathrm{C}$ for $24 \mathrm{~h}$.
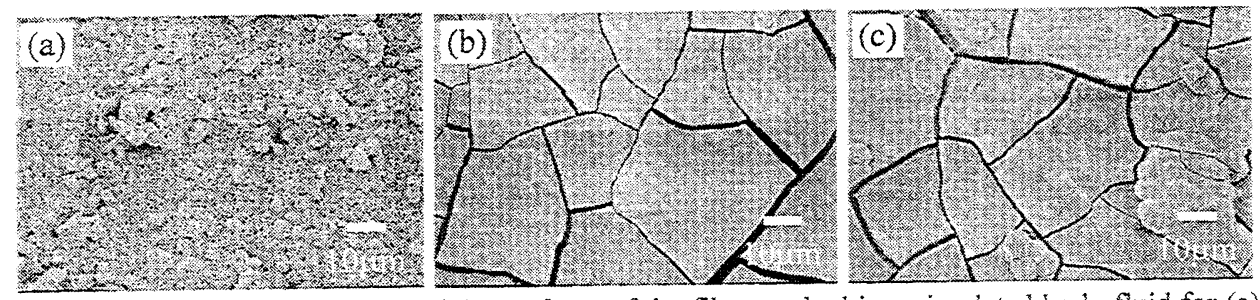

FIGURE 4. SEM photographs of the surfaces of the films soaked in a simulated body fluid for (a) 20 days, (b) and (c) 1 day. The films were sputtered from powder targets with Ca/P ratios of (a) 1.0 , (b) 1.5 , and (c) 1.67 .

\section{SUMMARY}

Polycrystalline hydroxyapatite thin films were successfully formed onto titanium metal 
plates by rf magnetron sputtering from three kinds of powder targets with 1.0, 1.5, and $1.67 \mathrm{of} \mathrm{Ca} / \mathrm{P}$ ratio, followed by hydrothermal annealing. The apatite-forming ability in a simulated body fluid increases with increasing $\mathrm{Ca} / \mathrm{P}$ ratio of the targets for sputtering. This is explained by changing not only crystallinities but also morphologies of the coatings.

\section{ACKNOWLEDGMENT}

This present work was partially supported by Grant-in-Aid for Encouragement of Young Scientists No. 10750499 from the Japanese Ministry of Education, Science, Sports and Culture. We thank Taihei Chemical Industry Co., Ltd. for providing all the calcium phosphate powders for sputtering targets in this work.

\section{REFERENCES}

1 T. Arashi, K. Yamashita, T. Kanazawa, T. Umegaki, Phos. Res. Bull., 1, 221 (1991).

2 K. Yamashita, T. Arashi, K. Kitagaki, S. Yamada, T. Umegaki, J. Am. Ceram. Soc., 77, 2401 (1994)

3 K. Yamashita, T. Yagi, T. Umegaki, J. Am. Ceram. Soc., 79, 3313 (1996).

4 K. Yamashita, T. Yagi, K. Nakamura, T. Umegaki, Phos. Res. Bull, 6, 123-126 (1996).

5 K. Yamashita, T. Yagi, J. Hamagami, T. Umegaki, Bioceramics, 2,337 (1996).

6 J. Hamagami, K. Nakamura, Y. Sekine, K. Yamashita, T. Umegaki, Bioceramics, 10, 207 (1997).

7 J. Hamagami, D. Kokubu, T. Umegaki, K. Yamashita, Bioceramics, 11, 219 (1998).

8 K. Yamashita, M. Matsuda, T. Arashi, T. Umegaki, Biomaterials, 19, 1239 (1998).

9 J. Hamagami, D. Kokubu, T. Umegaki, K. Yamashita, Korean J. Ceram., 4, 372 (1998).

10 J.E.D. Hulshoff, K. van Dijk, J.P.C.M. van der Waerden, J.G.C. Wolke, W. Kalk, J.A. Jansen, J. Biomed. Mat. Res., 31, 329 (1996).

11 T. Kokubo, H. Kushitani, S. Sakka, T. Kitsugi, T. Yamamoto, J. Biomed. Mater. Res., 24, 721 (1990). 